Thomas Faist

Universität Bielefeld

\title{
Transnationale Migration als relative Im- mobilität in einer globalisierten Welt
}

Arbeitspapiere - Working Papers

No. 11, 2006 
Faist, Thomas: Transnationale Migration als relative Immobilität in einer globalisierten Welt, Bielefeld: COMCAD, 2006

(Working Papers - Center on Migration, Citizenship and Development; 11)

The COMCAD Working Paper Series is intended to aid the rapid distribution of work in progress, research findings and special lectures by researchers and associates of COMCAD. Papers aim to stimulate discussion among the worldwide community of scholars, policymakers and practitioners. They are distributed free of charge in PDF format via the COMCAD website.

The opinions expressed in the papers are solely those of the author/s who retain the copyright. Comments on individual Working Papers are welcomed, and should be directed to the author/s.

University of Bielefeld

Center on Migration, Citizenship and Development (COMCAD)

Postfach 100131

D-33501 Bielefeld

Email Thomas Faist: thomas.faist@uni-bielefeld.de

Homepage: http://www.comcad-bielefeld.de 
Inhalt

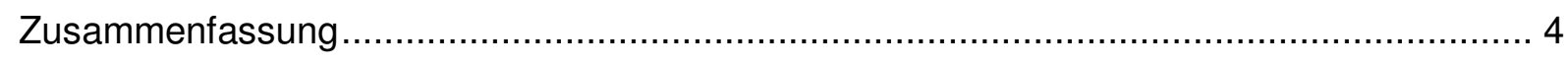

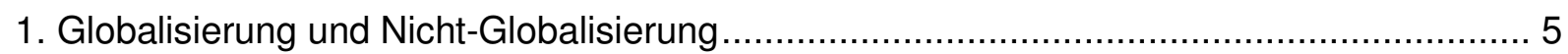

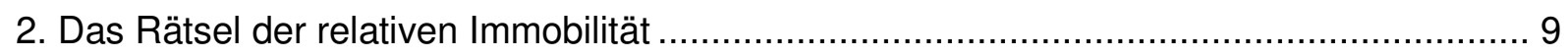

3. Transnationale Migration: Transnationalisierung und Universalisierung.........................25

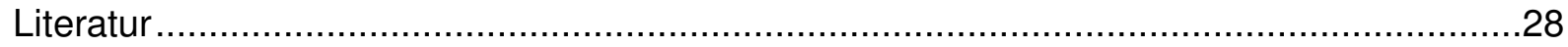

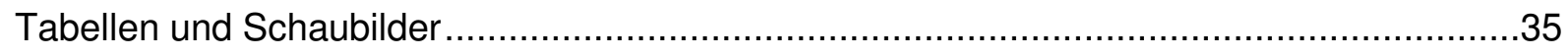




\section{Zusammenfassung}

Einerseits wird transnationale Migration angesichts der steigenden Zahl von Wandernden oft als ein Fall von Globalisierung interpretiert. Andererseits fällt auf, dass der Anteil von Migranten $^{1}$ an der Weltbevölkerung im letzten halben Jahrhundert kaum gewachsen ist. Allerdings übersehen beide Perspektiven das soziologisch zentrale Rätsel der relativen Immobilität: Warum gibt es so wenige transnationale Migranten aus den meisten Orten in den sogenannten Entwicklungsländern? Warum gibt es wiederum so viele Migranten aus einigen wenigen Orten des Südens in den Norden? Eine systematische Analyse der Faktoren für Immobilität und Mobilität ergibt, dass wichtige Ressourcen in der Regel lokal gebunden sind (local assets). Allerdings kann transnationale Migration angesichts der zunehmenden Mobilität von professionalisierten Fachkräften oder der zunehmenden Dichte von Informationsflüssen und Transportmöglichkeiten durchaus ein Beispiel für lebensweltlich geprägte Transnationalisierung begriffen werden, in dem primär lokal gebundene Ressourcen von Migranten über Staatsgrenzen hinweg mobilisiert werden.

\footnotetext{
${ }^{1}$ Aus Gründen der Vereinfachung des Lesens wird im Folgenden auf die weibliche Schreibform verzichtet. Die allgemein gehaltenen Personennennungen schließen jedoch sowohl Männer als auch Frauen mit ein.
} 


\section{Globalisierung und Nicht-Globalisierung}

Neben Fertilität und Sterblichkeit ist die räumliche Mobilität von Personen bekanntlich der dritte entscheidende Bestimmungsfaktor für die Bevölkerungsentwicklung. Für das Bevölkerungswachstum vieler OECD-Länder in den letzten Jahrzehnten spielte sogar Zuwanderung eine Hauptrolle, und nach vorliegenden Prognosen kann die rapide Alterung westlicher Industriegesellschaften durch Immigration zumindest verlangsamt und damit abgemildert werden (United Nations Secretariat 2001). Dabei wird die solchen Überlegungen zugrunde liegende transnationale Migration heutzutage von interessierten Beobachtern in unterschiedlicher Weise interpretiert.

Nach der einen Version, die als Mobilitätsszenario bezeichnet werden kann, hat transnationale Migration in den letzten Jahrzehnten kontinuierlich zugenommen und wird selbst bei abflachenden Kurven der Bevölkerungszunahme in den Entwicklungsländern für die reichen Länder ein immer größeres Kontrollproblem verursachen. Dieser Vorstellung gemäß tragen etwa gewaltige sozio-ökonomische Entwicklungsunterschiede zwischen reichen und armen Ländern, Kriege, ökologische Katastrophen, Klimawandel und vor allem auch Bürgerkriege zum wachsenden Migrationspotenzial bei. Auf den ersten Blick erscheint das Mobilitätsszenario plausibel. Die absolute Zahl transnationaler Migranten war um die Jahrtausendwende im Vergleich zur Mitte der 1960er Jahre tatsächlich beträchtlich höher (Tabelle 1): Die Zahl der außerhalb ihres Herkunftslandes lebenden Personen verdoppelte sich zwischen 1965 und 2005. Sie stieg von ca. 75 auf etwa 135 Millionen. $^{2}$ Insgesamt entspricht das Mobilitätsszenario der dominierenden Vorstellung von Globalisierung, dass alle grenzübergreifende Flüsse - Kapital, Güter, Dienstleistungen und Personen - in einer ähnlichen Weise einer Transnationalisierung unterworfen sind. Die dabei angenommene abnehmende Fähigkeit souveräner Nationalstaaten, den grenzübergreifenden Fluss beispielsweise von finanziellem Kapital zu kontrollieren, dient dabei auch als ein anschauliches Beispiel, das sinngemäß auf Migration übertragen wird (vgl. Castles und Miller 1993).

${ }^{2}$ Diese und alle folgenden Migrationsstatistiken beruhen auf äußerst groben Schätzungen. So erfassen manche Länder die Zahl der Ein- und Ausreisen (flows), andere wiederum diejenigen Immigranten bzw. Ausländer, die sich im Lande selbst aufhalten (stocks). Aber auch dabei schwankt die Bemessungsgrundlage. In Ländern wie Deutschland sind es alle diejenigen, die sich mindestens drei Monate aufhalten, also etwa auch Saisonarbeiter. In anderen Staaten wiederum, wie etwa Großbritannien, werden nur diejenigen erfasst, die schon mindestens ein Jahr dort leben. Dabei werden illegale bzw. irreguläre Migranten in keinem der Fälle dokumentiert. Als weitere Schwierigkeit einer realistischen Schätzung kommt hinzu, dass viele Länder, insbesondere in Afrika, gar keine Statistiken über Grenzübertritte und Immigranten führen (vgl. Bilsborrow et al. 1997). 
Eine dieser Interpretation entgegengesetzte Perspektive, der Immobilitätsansatz, ist weitaus vorsichtiger. Diese Sichtweise reduziert die angebliche Bedrohung durch transnationale Migration mit dem Hinweis darauf, dass gegenwärtig nur etwa drei Prozent der Weltbevölkerung außerhalb der Grenzen ihres jeweiligen Herkunftslandes leben (siehe Tabelle 2). Obwohl die Gesamtzahl der Migranten anstieg, ist somit seit Mitte der 1960er Jahre aufgrund des Bevölkerungswachstums der relative Anteil transnationaler Migranten an der Weltbevölkerung kaum gestiegen. Und auch in vergleichender historischer Perspektive ist die heutige Kettenund Massenmigration, die durch eine Zunahme von 2 bis 4 Millionen Migranten pro Jahr charakterisiert ist, also keineswegs beispiellos hoch. So war der relative Umfang freiwilliger transnationaler Migration im Europa des 20. Jahrhunderts und zu Beginn des 21. Jahrhunderts voraussichtlich sogar geringer als im 19. Jahrhundert (vgl. Segal 1993). Insgesamt verweist der Immobilitätsansatz darauf, dass offenbar nicht alle Produktionsfaktoren im gleichen Maße Globalisierungs- bzw. Transnationalisierungsprozessen ausgesetzt sind. Aus dieser Sicht gerät die transnationale Mobilität von Personen zu einem Fall von NichtGlobalisierung. Souveräne Staaten, zumindest diejenigen des Nordens, scheinen nach wie vor in der Lage, ihre Grenzen in dieser Hinsicht wirksam zu kontrollieren (Faist und Ette 2007; vgl. Torpey 2000) - auch wenn irreguläre Übertritte durchaus ein Grenzkontrollproblem darstellen.

Das sich aus diesen beiden grundverschiedenen Interpretationen ergebende Problem führt zum Rätsel der relativen Immobilität. Immobilität steht deshalb im Mittelpunkt, weil nur ein kleiner Teil der potenziellen Migranten tatsächlich transnational migriert; also das Migrationspotenzial um ein vielfaches höher ist als die Zahl bzw. der Anteil der tatsächlichen Migranten. Unter potenziellen Migranten sollen hier solche verstanden werden, die Motive und Gelegenheiten zur transnationalen Migration haben. Als relativ kann diese transnationale Immobilität deshalb gelten, weil es erstens in bestimmten Fällen von transnationaler SüdNord-Migration durchaus zu Massenmigration kommt und zweitens Menschen auch innerhalb ihrer Herkunftsländer wandern, beispielsweise im Rahmen der Land-Stadt-Migration. So ist etwa die Mobilität innerhalb Chinas viel höher als die transnationale Migration aus China in andere Länder.

Zweifelsohne fördern nun Globalisierungsprozesse transnationale Migration. So zum Beispiel, wenn unter Globalisierung etwa die grenzübergreifende Dynamik von Informationsflüssen oder günstigen Transportmöglichkeiten verstanden wird. Die massenmediale Verbreitung von Migrationsgelegenheiten, Anwerbung durch Immigrationsländer und erschwingliche Transportkosten gehören sicherlich zu den Faktoren, die transnationale Migration begünstigen (Eurostat 1995). Globalisierung spielt auch eine Rolle, wenn wir die zunehmende Uni- 
versalisierung von Meta-Prinzipien wie Menschenrechte oder Demokratie als Grundlage legitimer politischer Herrschaft nehmen. Dies spiegelt sich etwa in der „dritten Welle“ der Demokratisierung seit Ende der 1980er Jahre wider (Huntington 1991). ${ }^{3}$ In autoritären Systemen gibt es formal gesehen aufgrund von Ausreisebeschränkungen weniger Möglichkeiten zur geographischen Abwanderung als in demokratischen Systemen. Demokratische bzw. quasidemokratische Systeme hingegen errichten in der Regel zumindest keine massiven Ausreisebeschränkungen für ihre eigenen Bürger. Das läßt sich nicht nur an osteuropäischen Staaten seit Ende der 1980er Jahre durch die Aufhebung der "closed borders" in Europa nach dem Ende des Ost-West Konfliktes, sondern auch im Süd-Nord Kontext beobachten (Dowty 1987). Umgekehrt wirkt Globalisierung auch auf die Verregelung von Migration durch und in Immigrationsstaaten. Liberal-demokratische politische Systeme sind völkerrechtlich an internationale Regime wie die Genfer Flüchtlingskonvention und die humanitären Regeln des Familiennachzugs gebunden. Der Mechanismus internationaler Regime - also zwischenstaatlich verbindliche Regeln und Normen - wirkt insbesondere bei einmal in Gang gekommenen Migrationsbewegungen dahingehend, dass Immigrationsstaaten ihre völkerrechtlichen Verpflichtungen im Hinblick auf Menschenrechte nicht unilateral und abrupt zu ändern vermögen (Hollifield 1992). ${ }^{4}$

Allerdings wirken Globalisierungsprozesse nicht gleichmäßig, weder auf transnationaler noch auf subnationaler Ebene (Held et al. 1999). So sind etwa Süd-Nord Migrationsquoten vom subsaharischen Afrika nach Europa um einiges geringer als vom mediterranen Nordafrika nach Südeuropa (Hammar et al. 1997: 7). Eine nähere Analyse von derzeit wichtigen Emigrationsländern - wie etwa Mexiko, die Türkei oder die Philippinen - ergibt sofort, dass es in diesen Staaten auf subnationaler Ebene Regionen gibt, die bei etwa ähnlichem wirtschaftlichen Entwicklungsgrad und politischer Freiheit ganz verschiedene transnationale Migrationsquoten aufweisen (u.a. Eurostat 2000).

${ }^{3}$ Die erste Demokratisierungswelle begann in Amerika im frühen 19. Jahrhundert. An ihrem Ende standen rund 30 mehr oder minder entwickelte demokratische Regime. Die zweite Welle setzte mit der Demokratisierungspolitik der Alliierten nach dem Zweiten Weltkrieg in den besiegten Staaten Deutschland, Italien und Japan ein. Weitere Schubkraft erfuhr Demokratisierung durch die Dekolonialisierung der 50er und 60er Jahre des 20. Jahrhunderts. Die dritte Demokratisierungswelle kam Mitte der 1970er Jahre. Auf ihr befanden sich viele autoritäre oder halbautoritäre Regime in Südeuropa, Lateinamerika und Ostasien. Hinzu kam Ende der 1980er und zu Beginn der 1990er Jahre eine vierte Welle. Sie erfasste unter anderem die mittel- und osteuropäischen Staaten, die nach dem Zerfall der östlichen Bündnisse und der UdSSR den Übergang zu demokratischen Formen der Herrschaft antraten.

${ }^{4}$ Wichtige Immigrationsländer in Regionen wie dem Mittleren Osten (z.B. Saudi-Arabien und Kuwait) halten internationale Konventionen zum Schutz von Migranten und deren Angehörigen in der Regel in weniger hohem Maße ein. 
Die hier verfolgte Fragestellung zielt daher darauf ab, zu erklären, warum nur relativ wenige der potenziellen Migranten tatsächlich über Grenzen hinweg wandern. Davon unberührt bleiben erst einmal Einschätzungen, die diese Wanderungsbewegungen als vernachlässigenswert gering und sogar für ökonomisch-demographische Zwecke als wünschenswert ansehen (z.B. viele der Beiträge in Bade und Münz 2002) oder als neue, Unheil stiftende Völkerwanderungen apostrophieren, die einstmals kulturell homogene Nationalgesellschaften multiethnisch fragmentieren und angesichts des vermeintlich geringen kulturellen Kapitals neuer Migranten eher Probleme für Arbeitsmarkt und soziale Sicherungssysteme erwarten (Birg 2001). Die geeigneten Fragestellungen wären demnach nicht: Warum gibt es so viele transnationale Süd-Nord-Migranten? Oder: Warum gibt es so wenige transnationale Migranten? Die soziologisch relevanten Fragen sind hingegen: Erstens, warum gibt es so wenige Migranten aus den meisten Regionen des Südens in den Norden? Und zweitens: Warum gibt es so viele Migranten aus so wenigen Orten des Südens in relativ wenige im Norden? Sowohl die Mobilitäts- als auch die Immobilitätsthese transnationaler Migration - und damit auch die Globalisierungs- und die Nicht-Globalisierungsthese - sind nur teilweise zutreffend und müssen durch eine differenziertere Interpretation ersetzt werden.

Die Analyse kann dabei an zwei plausiblen Vermutungen ansetzen: Erstens lohnt es sich, nicht ausschließlich makro-strukturelle Ursachen transnationaler Süd-Nord-Migration, wie beispielsweise sozio-ökonomische Ungleichgewichte zwischen Süd und Nord sowie politische Unterdrückung in den Herkunftsregionen zu untersuchen. Stattdessen gilt es auch zu fragen, welche Ressourcen aktiviert werden müssen, damit aus potenziellen Migranten tatsächliche Migranten werden. Die teilweise große Kluft zwischen potenziellen und tatsächlich Handelnden ist auch in der Forschung zu sozialen Bewegungen ein altbekanntes Problem (McPhail und Miller 1973). Dabei geht es bei transnationalen Migranten nicht nur um den Transfer ökonomischen Kapitals in Form von Geld oder kulturellen Kapitals in Form von Qualifikationen wie Bildungsabschlüssen. Vielmehr spielt insbesondere auch der Transfer von sozialen und symbolischen Bindungen - also gebundene Transaktionen zwischen Personen und Kollektiven - eine große Rolle. Soziale und symbolische Bindungen sind ein typisches Beispiel für lokale, d.h. ortsgebundene Ressourcen. Diese können aber unter bestimmten Bedingungen wie Pioniermigration, Netzwerke und institutionalisierten Anwerbungen „transportiert“ werden. Daher ist soziale Nähe nicht notwendigerweise an geographische Nähe gebunden (Simmel 1992: Teil IX). Zweitens ist genau aus diesem Grunde transnationale Migration vom Austausch von Gütern, Kapital und Dienstleistungen zu unterscheiden. Grenzübergreifende räumliche Mobilität geht mit lebensweltlichen Bindungen von Mobilen und Sesshaften einher. Transnationale Mobilität beruht neben Globalisierung im Sinne einer Zunahme der absoluten Zahl transnationaler Migranten und der Universalisierung von Ab- 
wanderungs- und teilweise auch Zuwanderungsmöglichkeiten vor allem auch auf der Transnationalisierung primär lokaler Bindungen, Ressourcen und Deutungen. Da es sich hierbei vorwiegend um Bindungen nicht-staatlicher Akteure handelt, ließe sich am ehesten von regional begrenzten Transnationalisierungen sprechen. ${ }^{5}$ Transnationalisierung impliziert im Falle transnationaler Migration dabei keineswegs notwendigerweise auch die generelle zunehmende Öffnung staatlicher Grenzen für die Wanderung von Personen.

Zunächst wird das Rätsel relativer Immobilität geklärt, also der Frage nachgegangen, warum es so viele und so wenige Migranten gleichzeitig gibt. Dann wird in einem zweiten Schritt gefragt, wie die Perspektiven von Globalisierung und Nicht-Globalisierung bzw. des Mobilitäts-und Immobilitätsszenarios durch eine adäquatere Betrachtungsweise ergänzt werden können.

\section{Das Rätsel der relativen Immobilität}

Es gibt fünf Hauptursachen für den hohen Grad von Immobilität im Bereich transnationaler Süd-Nord-Migration, die zur Beantwortung der Frage, warum es so wenige Migranten aus den meisten Regionen des Südens in den Norden gibt, beitragen. Dabei wird unter Migration im Folgenden sowohl Arbeitsmigration und Fluchtmigration, relativ freiwillige Wanderung mit hohen Freiheitsgraden und erzwungene Abwanderung verstanden. Leider übernimmt die sozialwissenschaftliche Analyse in der Regel unhinterfragt die verwaltungsmäßige und juristische Differenzierung von Arbeitsmigranten und Asylbewerbern, ohne die fundamentalen Gemeinsamkeiten zu berücksichtigen. Eine sinnvollere Unterscheidung für die Analyse von Ursachen und Dynamik transnationaler Migration verläuft entlang der Freiheitsgrade potenzieller Migranten, etwa solchen mit hohen Graden an Entscheidungsfreiheit, worunter auch Flüchtlinge fallen können, die antizipierend wandern (Kunz 1973) und solche mit geringen Wahlmöglichkeiten, die durch physische oder strukturelle Gewalt signifikant eingeschränkt sind.

Alternativen zu transnationaler Süd-Nord-Migration: Binnenmigration und Süd-Süd-Migration Binnenmigration innerhalb der Entwicklungsländer ist in vielen Fällen eine praktikable Alternative. Aber auch wenn Binnenmigration eine Alternative zu transnationaler Arbeitsmigration

${ }^{5}$ Der Begriff „international“ bezieht sich auf die Beziehungen zwischen Staaten, der Begriff „transnational“ auf grenzübergreifende Beziehungen, in denen hauptsächlich auch nicht-staatliche Agenten bzw. Akteure beteiligt sind. 
und Flüchtlingsbewegungen darstellt, so ist sie häufig als Stufenmigration der erste Schritt in Richtung grenzüberschreitender Wanderung, allerdings nicht unbedingt in die Industrieländer (OECD 2000). Denn es gibt Migrationsströme (flows) zwischen verschiedenen Staaten des Südens in beträchtlichem Umfang: Mehr als die Hälfte aller transnationalen Migranten aus dem Süden wanderte Ende der 1980er Jahre und in den 1990er Jahren von einem Entwicklungsland in ein anderes und nicht in die entwickelten Industrieländer. Süd-SüdMigrationsströme sind dem Anteil nach also bedeutsamer als Süd-NordMigrationsbewegungen (Tabellen 1 und 2). Bezogen auf Flüchtlinge sind es gar weltweit geschätzte $97 \%$, die in ihren Herkunftsländern als „displaced persons“ bleiben oder in andere Entwicklungsländer wandern.

Ein ähnliches Bild ergibt sich, wenn man sich auf den Anteil der Migranten (stocks) an der Weltbevölkerung konzentriert. Die Mehrzahl der transnationalen Migranten konzentriert sich auf die Entwicklungsländer (Tabelle 1). Und zu Anfang des 21. Jahrhunderts lebten über die Hälfte der Flüchtlinge und Asylbewerber auf der Welt im Mittleren Osten und Südasien. Dabei machten zwei Gruppen, Palästinenser und Afghanen, allein 40\% des weltweiten Flüchtlingsanteils aus. Dabei ist auf das Ziel der Flüchtlinge bezogen ein wohl kaum vermutetes Land Spitzenreiter, nämlich der Iran, in dem fast ein Viertel der weltweit mehr als 20-30 Millionen offiziell registrierten Flüchtlinge leben (UNHCR 2006).

\section{(1) Verelendung}

Das Ausmaß absoluter Deprivation durch materielle Armut beschränkt in einschneidender Weise den Grad jeweiliger Wahlfreiheit potenzieller Migranten. Ende der 1990er Jahre lebte ca. ein Fünftel der Weltbevölkerung in absoluter Armut (UNDP 2003). Dies bedeutet, dass es vielen Menschen in bestimmten Regionen des Südens schlicht an Ressourcen nicht nur für geplanten politischen Widerspruch (voice), sondern auch für geographische Abwanderung im Inland oder gar über die Staatsgrenzen hinaus (exit) mangelt (vgl. Hirschman 1970 zur Begrifflichkeit). Sie verharren in einem Zustand der Resignation inmitten einer feindseligen Umgebung. So fehlen beispielsweise den meisten Menschen im Afrika südlich der Sahara die grundlegenden Mittel, um vom Süden bis in die OECD-Welt zu wandern. In Fällen solch starker struktureller Beschränkungen können die betroffenen Personen strenggenommen nicht einmal mehr als potenzielle Migranten betrachtet werden. Es ist hier nicht überraschend, dass für einen beträchtlichen Anteil vertriebener und verfolgter Personen nur kurzfristige Flucht in ein Nachbarland möglich ist. Die Opfer der Bürgerkriege in Ruanda und Burundi der 1990er Jahre oder neuerdings in der Region Dhafur im Sudan können hier als anschauliche Beispiele dienen. 
(2) Lokale Ressourcen: kulturelles, soziales und symbolisches Kapital

Viele Ressourcen potenzieller Migranten sind an bestimmte Orte („place“) bzw. Regionen gebunden. Zu solchen Ressourcen zählen ökonomisches Kapital wie Geld und Grundbesitz, kulturelles Kapital wie schulische Zeugnisse und berufliche Qualifikationen und nicht zuletzt soziales Kapital wie Ressourcen in familiären und weiteren Gruppenbindungen sowie symbolisches Kapital wie gemeinschaftlich geteilte Symbole. Ein bestimmter Anteil der Ressourcen und Fähigkeiten von Personen und Kollektiven sind insofern ortspezifisch, weil sie allein in einer bestimmten abgegrenzten Region oder Land in vollem Maß genutzt und nicht ohne Weiteres zu Aufenthaltsorten ins Ausland transferiert werden können. Dies verweist auf die grundsätzlichen faktischen Probleme der grenzüberschreitenden Transferierbarkeit erworbener Fähigkeiten und akkumulierter Ressourcen. Beim Transfer von kulturellem Kapital ist etwa zu beobachten: Auch wenn globale Arbeitsmärkte für bestimmte hochqualifizierte Berufsgruppen existieren, so etwa für Manager, Experten der Informationstechnologie, Wissenschaftler und so genannte „professionals“ im weiteren Sinne, gibt es gleichwohl länderspezifische staatliche oder berufsständische Zulassungsverfahren und damit entscheidende Hürden für die vollständige Anerkennung im Ausland erworbener Qualifikationen (Kuptsch und Fong 2005).

Als eine Alternative zu Migration können potenzielle Migranten durch ortspezifische Vorteile (sogenannte „insider advantages“) die Früchte relativer Sesshaftigkeit ernten. Sprachkenntnisse sind ein wichtiges Beispiel für kulturelles Kapital, die einen Vorteil für die Kenner der ortsüblichen Verhältnisse darstellen. Sprache ist ein unerlässliches Mittel zur Kommunikation, mittels derer wiederum diverse Ressourcen zu erwerben sind. Die Wanderung in ein anderes Land dagegen verursacht zumeist die Kosten des Erwerbs einer neuen Sprache. Solange ein Immigrant die Sprache des Immigrationslandes nicht wenigstens zu einem gewissen Grad beherrscht bzw. bereit ist, diese relativ schnell zu erlernen, existieren schwerwiegende Hürden im Zugang zu erstrebenswerten Arbeitsplätzen, zu beruflicher Selbstständigkeit und zu adäquaten Wohnungen.

Um über das von der Ökonomie auf Humankapital (bzw. kulturelles Kapital) angewandte Prinzip der „insider advantages“ hinauszugelangen, ist ein Blick auf die Merkmale von sozialem und kulturellem Kapital in sozialen und symbolischen Bindungen notwendig. Ein umfassender Kapital-Ansatz erlaubt es uns, nicht nur zwischen starken und schwachen Bindungen zwischen Personen zu unterscheiden, sondern auch systematisch die in den sozialen und symbolischen Bindungen zwischen Personen vorhandenen Ressourcen zu thematisieren. Dabei hilft eine erweiterte Netzwerkmethodologie weiter. Netzwerkansätze sind Methoden, die Handeln primär aus der strukturellen Position von Akteuren bestimmen (White et al. 1976). Der Schwerpunkt liegt dabei auf der Syntax sozialer und symbolischer Bindungen. 
Theoretische Aussagen über das Verhältnis der Akteure geraten allenfalls implizit in diese Methodologie hinein (Granovetter 1979: 501). Bildlich gesprochen lässt sich der Netzwerkansatz erweitern, indem die Grammatik sozialer und symbolischer Bindungen über die Syntax hinaus durch eine Semantik ergänzt wird. Dies kann durch den Kapital-Ansatz erfolgen, der die in Netzwerktransaktionen vorfindbaren Ressourcen wie Reziprozität als Tausch, Reziprozität als soziale Norm und Solidarität als Grundformen thematisiert (Faist 2000b: Kapitel 1).

Die unterschiedlichen Formen sozialen und kulturellen Kapitals sind in sozialen und symbolischen Bindungen eingebettet. Soziale Bindungen stellen eine kontinuierliche Serie interpersonaler Transaktionen dar, denen die Beteiligten gemeinsame Interessen, Verpflichtungen, Erwartungen und Normen zuschreiben. Symbolische Bindungen sind im Unterschied dazu kontinuierliche Transaktionen, die direkt oder indirekt stattfinden können und an welche die Beteiligten gemeinsame Bedeutungszuschreibungen, Erinnerungen und Zukunftserwartungen knüpfen. Symbolische Bindungen können über unmittelbare Beziehungen zwischen Personen hinausgehen, indem sie sich allgemeiner an Mitglieder desselben Glaubens, derselben Sprache, Ethnizität oder gar Nationalität richten.

Soziales Kapital bezeichnet die in sozialen Bindungen inhärenten Ressourcen, die es Personen erlauben, in Netzwerken, Gruppen und Organisationen miteinander zu kooperieren. Soziales Kapital stellt eine Reihe von Mechanismen dar, die zu einer Stärkung oder, im Falle seiner Abwesenheit, zur Schwächung von Kooperation führt (Putnam 1993: Kapitel 6).

Wir können die folgenden Formen sozialen Kapitals unterscheiden, die als Mechanismen der Vernetzung wirken. Einmal handelt es sich bei Reziprozität um ein Merkmal des sozialen Tausches: Hier geht es um gegenseitige Verpflichtungen und Erwartungen der Akteure, die mit bestimmten sozialen Bindungen assoziiert werden und die auf einem in der Vergangenheit geleisteten Austausch oder entsprechenden Dienstleistungen basieren (Coleman 1990: 306-9). Solche Verpflichtungen und Erwartungen können Ergebnis zweckrationaler Handlungen sein, wie dies beispielsweise in der Anwendung des „tit-for-tat“-Prinzips („Wie du mir, so ich dir") zum Ausdruck kommt. Zum anderen kann Reziprozität als soziale Norm gefasst werden: Wenn die eine Partei etwas von der anderen erhält, erfordert dies einen Ausgleich (Gouldner 1960: 160). Dieser kann mit zeitlicher Verzögerung geschehen und unter Gleichen bzw. Ungleichen vollzogen werden.

Symbolisches Kapital bezeichnet im Unterschied zu sozialem Kapital die in symbolischen Bindungen vorfindbaren und darüber mobilisierbaren Ressourcen. Es bindet durch kollektive Gefühle der Zugehörigkeit Personen an Netzwerke, Gemeinschaften und Organisationen. 
Bei symbolischen Kapital handelt es sich um gemeinsame Deutungsmuster, die als Ressourcen wie Solidarität in symbolischen Bindungen zum Tragen kommen, z.B. Wir-Gefühle in einer Verwandtschaftsgruppe (in Anlehnung an Max Webers Charakterisierung ethnischer Gruppen, Weber 1980: 536-7). Darunter fallen Ideen, Anschauungen, Wertungen und Symbole, die einen gemeinsamen Referenzrahmen oder gar Lebenskontext für eine Gruppe bilden. Ein wichtiges Element ist ein geteilter Code oder eine gemeinsame Sprache. In ihrer idealtypischen Form finden wir Deutungsmuster in kulturellen Gemeinschaften wie Familien, ethnische Gruppen, religiöse Gemeinden und Nationen. Beziehen sich die Deutungsmuster auf hochkomplexe Aggregate wie Heimat oder Nation, dann handelt es sich um „kollektive Repräsentationen“ (Durkheim 1965: 471). Deutungsmuster und spezielle Ausprägungen wie kollektive Repräsentationen ermöglichen Empathie und bilden damit die Grundlage für Solidarität mit anderen in einer Gruppe, die ähnliche Positionen oder Standpunkte einnehmen wie man selbst (Portes 1995: 15) oder mit denen man sich über symbolische Bindungen verknüpft fühlt. Solidarität bezieht sich auf expressive Formen symbolischer Transaktionen. Transaktionen, die auf den Mechanismen von Reziprozität und Solidarität beruhen, können allerdings erwünschte und unerwünschte Folgen haben. Sie vermögen nicht nur Kooperation zu erhöhen, sondern auch die individuelle Freiheit von Personen auf unterschiedliche Weise einzuschränken. Kurz, sie fungieren teilweise als „negatives Kapital": Die Norm der Reziprozität kann zu erzwungener Migration oder Immobilität führen.

Soziales und symbolisches Kapital sind immer gleichzeitig individuelle und kollektive Ressourcen. Sie ermöglichen es zum einen Personen, ihre Interessen in Netzwerken und Kollektiven zu verfolgen (Bourdieu 1983) und zum anderen Kollektiven, Personen in eine Gruppe zu integrieren und ihrer Kontrolle zu unterwerfen. Soziales, symbolisches und teilweise kulturelles Kapital und vor allem die Mechanismen Reziprozität und Solidarität sind in Bezug auf Mobilität ambivalente Ressourcen. Es sind lokal gebundene Ressourcen, deren Wert auf bestimmte soziale Räume wie Gemeinden und Regionen beschränkt und nicht einfach über Staatsgrenzen hinweg transferierbar ist. Potenzielle Migranten stehen angesichts der Transferierbarkeit dieser Ressourcen vor zwei Problemen: der Aufrechterhaltung von Bindungen im Emigrationskontext und den Adaptations- bzw. Integrationskosten im Immigrationsland. Erstens erhöht die Pflege von sozialen und symbolischen Bindungen die anfallenden Transaktionskosten, also diejenigen Kontrollkosten, die für die Einhaltung von Abmachungen aufgewendet werden müssen (Williamson 1981). Räumliche Distanz erhöht die jeweiligen Transaktionskosten, weil etwa arbeitsteilige Abmachungen wie Rücküberweisungen der Migranten vs. Haushalts- und Kinderarbeit der Zurückgebliebenen schwer einzufordern und zu überwachen sind. Ein Beispiel anhand der geschlechtlichen Arbeitsteilung aus den Philippinen mag diese Schwierigkeit verdeutlichen (Hugo 1981): Es ist nicht von vornherein aus- 
gemacht, dass bei transnationaler Migration der Mütter als Krankenschwestern oder zur Arbeit in Haushalten der Golfstaaten sich neben den Großmüttern auch die zurückgebliebenen Väter entscheidend um die Kindererziehung bemühen. Falls aber die Kindererziehung bei temporärer geographischer Abwesenheit der Mütter nicht geregelt ist, kann es keine reziproke Arbeitsteilung zwischen der Wanderarbeit der Mütter einerseits und der Kinderarbeit der Väter und der Großeltern andererseits geben. Dazu bedarf es entweder Rollen- bzw. Verhaltensänderungen zwischen den Geschlechtern und/ oder eines weitläufigen familiären Unterstützungssystems. ${ }^{6}$ Zweitens sehen sich transnationale Migranten häufig hohen Eingliederungskosten im Ausland gegenüber - beispielsweise dem Erlernen einer neuen Sprache, dem Aufbau neuer sozialer Beziehungen und gemeinschaftlicher Bindungen. Diese Integrationskosten können insbesondere für Pioniermigranten mit geringen formalen Bildungsabschlüssen extrem hoch sein. Falls nicht schon Kolonien von Migranten im Immigrationsland existieren, die mit Hilfe einer vertrauten Sprache, Selbsthilfegruppen, kulturellen Angeboten und vor allem auch Kontakten in Arbeits- und Wohnungsmärkten einen gleitenden Übergang erlauben, erscheinen die Hürden für Neuankömmlinge oft unüberwindbar.

Je öfter nun ein Migrant entschieden hat, nicht zu migrieren, desto höher ist die Wahrscheinlichkeit, dass er oder sie immobil bleibt. Dieses auf Immobilität angewandte Prinzip der kumulativen Kausalität hilft, Verbindungen zwischen systemspezifischen Beobachtungen zu schaffen. In der Terminologie der Kybernetik würde man dabei von positiven Rückkoppelungssystemen sprechen (Mayurama 1963: 175). Kumulativ ist die Kausalität insofern, als auf jeder Stufe der vorhandene dominante Effekt noch verstärkt wird, entweder in Richtung auf Immobilität oder in die gegenläufige Richtung auf Mobilität. Bei Immobilität beobachten wir beispielsweise in vielen Entwicklungsländern einen hohen Anteil potenzieller Migranten angesichts verbreiteter Landknappheit. Dabei ist auffällig, dass in manchen der betroffenen Regionen landwirtschaftliche oder Handwerks-Kooperativen (Kyle 2000) entstehen und sich durchsetzen. Diese treiben beispielsweise die Vermarktung von Produkten voran und fördern durch soziale und wirtschaftliche Erwartungssicherheit vor Ort, also in situ Adaptation. Solche Kooperativen fördern Immobilität, die wiederum Initiativen zur Stärkung vorhandener oder der Gründung neuer kollektiver Zusammenschlüsse befördert. Obwohl diese Form ökonomischer Organisation kurzfristige räumliche Mobilität wie etwa Geschäftsreisen fördern

${ }^{6}$ Dabei ist natürlich die Einsicht aus der Anthropologie zu beachten, dass unter dem Begriff Familie in verschiedenen Regionen der Welt unterschiedliche soziale Formationen verstanden werden - von Abstammungsgemeinschaften bis hin zu „fiktiven“ Verwandtschaftsbeziehungen (Kibria 1993). 
kann, sinkt - ceteris paribus - tendenziell der Anteil der Personen, die transnationale Migration als Strategie für sozio-ökonomische Mobilität nutzen. ${ }^{7}$

\section{(4) Kurzfristige Zeit-Raum Strategien: Saisonale Migration}

Gerade die lokale Gebundenheit von sozialem und symbolischem Kapital führt dazu, dass relativ kurzfristige Zeit-Raum-Strategien von Personen verwendet werden, die ansonsten relativ sesshaft sind (Hägerstrand 1975). Diese Personen transferieren beispielsweise ihr kulturelles Kapital wie Bildungszertifikate ins Ausland, während das jeweilige soziale und symbolische Kapital überwiegend im Herkunftsland verbleibt. Über Jahrhunderte hinweg haben transnationale Migranten als saisonale Landarbeiter, Haushaltshilfen und Bauarbeiter gearbeitet und wirken neuerdings vermehrt auch als Ärzte, Krankenschwestern, Wissenschaftler, Computerspezialisten und Wirtschaftsmanager. Viele weitere Berufe eignen sich für eine befristete Tätigkeit im Ausland. Heutzutage arbeiten mehr Personen auf Basis befristeter Visa und Arbeitserlaubnisse außerhalb ihres Herkunftslandes, als es dauerhafte Einwanderer gibt. Als Beispiel mögen auch viele Angestellte multinationaler Unternehmen dienen (Findlay 2003). Dies wäre vielleicht ein Merkmal, das sich in der Tat mit dem Begriff eines neuen Schubes an Globalisierung im Sinne der Transnationalisierung des Produktionsfaktors Arbeit seit den 1970er Jahren assoziieren ließe. Andererseits ist zu beachten, dass die europäischen und asiatischen Arbeitsmärkte im 19. Jahrhundert wahrscheinlich in noch größerem Maße durch temporäre Migration gekennzeichnet waren als dies heute der Fall ist (Strikewerda 1997). Dies hängt damit zusammen, dass die souveränen Nationalstaaten als Immigrationsstaaten ihre Anstrengungen hinsichtlich externer Kontrollen wie Pässe und Visa und interner Kontrollen wie Arbeitserlaubnisse in den letzten hundert Jahren erheblich verstärkt haben. Dazu trug auch die Entwicklung ausgeprägter Wohlfahrtsstaaten in der heutigen OECD-Welt bei, die den Schutz von einheimischen Arbeitskräften impliziert (so genann-

\footnotetext{
${ }^{7}$ Aus dieser Interpretation kann jedoch nicht voreilig geschlossen werden, dass ökonomische Entwicklung per se die Migrationsquoten senken würde. Vielmehr verweist das vorliegende Beispiel auf ein Wachstum an Erwartungssicherheit, das auch mit wirtschaftlicher Entwicklung einhergeht. Schon die Klassiker der sozialrelationalen Migrationsforschung wiesen darauf hin, dass Mechanismen wie Kooperativen und Genossenschaftskassen Immobilität fördern (Thomas/ Znaniecki 1927, Bd. 4: 178-304). Von diesem Argument zu unterscheiden sind einfältige Vorschläge, die in Politiken wie dem sog. root causes approach zum Ausdruck gebracht werden. Demzufolge soll wirtschaftliche Entwicklung vor Ort in Emigrationsregionen dafür sorgen, dass der Migrationsdruck auf die OECDLänder abnimmt (vgl. Appleyard 1992). Der tatsächliche Zusammenhang zwischen ökonomischer Entwicklung ist jedoch weitaus problematischer und kann in stilisierter Weise kurz und bündig in der sog. umgekehrten U-Kurve gefaßt werden (Faini/ Venturini 1994): Es besteht eine in Glockenform darstellbare Korrelation zwischen der Migrationsquote einerseits und dem wirtschaftlichen Entwicklungsgrad, gemessen am Pro-Kopf-Einkommen, andererseits. Bei geringem BSP pro Kopf und bei sehr hohem ist die Migrationswahrscheinlichkeit sehr gering, bei mittlerem Einkommen relativ hoch. Derzeit liegt diese Zahl bei etwa US $\$ 4.000$ bis 5.000 und trifft somit am ehesten für Staaten wie die Türkei, Marokko, Mexiko und die Philippinen zu.
} 
tes „Inländerprimat"). Insgesamt könnte gerade das Beispiel der kurzfristigen Zeit-RaumStrategien von Personen zur Vermutung Anlass geben, daß es sich bei dem relativen Anstieg im Vergleich zu den 50er und 60er Jahren des 20. Jahrhunderts vor allem um einen neuen Schub an regionaler Globalisierung und nicht um ein neuartiges Phänomen handelt.

\section{(5) Alternativen zu Abwanderung: Loyalität und Widerspruch}

In der Vergangenheit wurde Immobilität als eine traditionelle Verhaltensweise gedeutet, so etwa in den modernisierungstheoretischen Schriften über den Mittleren Osten (u.a. Lerner 1958). Immobilität kann allerdings in einer angemesseneren Weise interpretiert werden und zwar als Folge von Loyalität, also als eine sozialen und symbolischen Bindungen inhärente Ressource. Loyalitäten von potenziellen Migranten zu bestimmten Kollektiven von Familien bis hin zu Nationen begünstigen bei Leistungsabfall Immobilität, wie z.B. in wirtschaftlichen oder politischen Krisen. Loyalität wirkt also tendenziell Abwanderung (exit) entgegen. Um die jeweiligen Lebenschancen zu verbessern, gibt es aus der Perspektive der potenzielIen Migranten bei ausgeprägter Loyalität zum politischen System Alternativen zur geographischen Abwanderung, wie beispielsweise politischer Widerspruch (voice). Widerspruch dient als politische Meinungsäußerung und politische Beteiligung im jeweiligen Herkunftsstaat. Hier treffen wir auf einen engen Zusammenhang von Bevölkerung und Volk: Die Loyalität der Bürger und Bürgerinnen entscheidet zentral über die Zusammensetzung der Bevölkerung und Bevölkerungsbewegungen eines souveränen Staates. Dabei wäre folgender Zusammenhang zu vermuten: Je höher die Loyalität der Mitglieder eines Volkes gegenüber dem politischen Regime und je stärker möglicherweise die Legitimität der Regierung, desto geringer - ceteris paribus - die Wahrscheinlichkeit von Abwanderung aufgrund von Unzufriedenheit bei politisch verursachtem Leistungsabfall. Umgekehrt sind die hohen, in der Regel unfreiwilligen Abwanderungsquoten bei Bürgerkriegen ein manifestes Beispiel für den Fall, dass Migranten in der Wahl der Optionen gerade nicht frei sind, d.h. ihre Entscheidungen nur geringe Freiheitsgrade aufweisen.

Genau genommen sind allerdings selbst bei transnationaler Migration Widerspruch (voice) und Abwanderung (exit) keine sich wechselseitig ausschließenden Optionen, sondern können und werden während transnationaler Migrationsprozesse sukzessive genutzt. Häufig geht politischer Widerspruch territorialer Abwanderung voraus, ergänzt sie oder folgt dieser. Als ein extremes Beispiel für die letzte Variante mögen hier palästinensische Kämpfer gelten - die „refugee warriors“ -, die gewaltsam für die politische Unabhängigkeit einer „Nation ohne Staat" eintreten (Zolberg et al. 1989). 
Die fünf genannten Gründe für Nicht-Migration stellen aber nur die eine Seite des Rätsels der relativen Immobilität dar. Unter günstigen Rahmenbedingungen können auch Kettenwanderungen aus ganz bestimmten Regionen des Südens in den Norden entstehen. Hier lautet also die Frage: Warum gibt es so viele transnationale Migranten aus so wenigen Regionen des Südens in ausgewählte Gebiete des Nordens? Dabei sind es vor allem zwei Faktoren, die eine Rolle spielen.

\section{(1) Einwanderungs- und Rekrutierungspolitiken der Immigrationsstaaten}

In der Regel wurden die bekannten Fälle der transnationalen Migration von Süd nach Nord seit dem Zweiten Weltkrieg durch dezidierte Anwerbepolitiken der Immigrationsstaaten selbst ausgelöst. Und oft folgte später dann die Richtung der Fluchtmigration den eingetretenen Pfaden der Arbeitsmigration. Die Richtung und die Ziele der gegenwärtigen transnationalen Migration sind insofern relativ eindeutig bestimmbar. Die dem Konzept Migrationssysteme zugrunde liegende Idee lautet, dass ein intensiver, wenn auch nicht symmetrischer Austausch von Gütern, Ideen und Kapital zwischen einem Set von Ländern mit Migration einhergeht. Dies bedeutet, dass regional differenzierte Systeme entstehen, in denen Kernstaaten als Immigrationsregionen und die Peripherien als Emigrationsregionen fungieren (vgl. Kritz et al. 1992). Der überwiegende Anteil der Süd-Nord-Migration stammt dabei entweder aus früheren Kolonien oder wirtschaftlich abhängigen Territorien des Südens und ist in die reichen Industrieländer des Nordens gerichtet. Ein Beispiel für den ersten Fall bilden Surinam und die Niederlande, für den zweiten Fall stehen das ehemalige Jugoslawien und die Bundesrepublik Deutschland. In beiden Fällen ging der Impetus zum „pull“ von den Ländern des Nordens aus, der die „push“-Faktoren im Süden erst mobilisierte. Es sind nicht nur die meisten transnationalen Migranten aus wenigen Regionen gekommen, sondern auch wiederum in wenige Regionen gewandert. Dies weist darauf hin, daß sich transnationale Migration in regional spezifischen, Länder und Regionen des Südens und Nordens umfassenden, Migrationssystemen vollzieht und dabei in klar definierbaren sozialen Formationen wie Migrationsnetzwerken verläuft.

Daraus sollte allerdings nicht voreilig der Schluß gezogen werden, dass das herkömmliche „push-pull-Modell“ zur Erklärung der Ursachen und Dynamik von Migration (Lee 1964) vom Ansatz der Migrationssystemtheorie und neuerdings von transnationalen Modellen abgelöst worden sei. Das wäre ein schwerwiegender Fehlschluss im Hinblick auf Analyseebenen. Das push-pull-Modell, auch wenn es eine empirisch erwiesenermaßen falsche Kausalität von „push“ zu „pull“ suggeriert, ist die konsequente Umsetzung der Theorien rationaler Wahl auf Bevölkerungsbewegungen (vgl. Esser 1980: 28) und hat neben sozialpsychologischen Erklärungsansätzen durchaus Erklärungskraft für die Mikro-Ebene. Hinter Migrationssystemtheo- 
rien wiederum verborgen sind sozialstrukturelle Ansätze, die von einer Makro-Ebene ausgehen und später auf einer Meso-Ebene um intermediäre Elemente wie die Dynamik von Netzwerkmigration (Massey et al. 1993) und neuerdings Ressourcen in sozialen und symbolischen Bindungen ergänzt werden. Eine Verbindung von systemorientierten, zumeist makrostrukturellen Ansätzen mit auf Sozialintegration abzielenden Meso-Theorien steht in der Migrationsforschung allerdings noch aus. ${ }^{8}$ Hier geht es im Folgenden um eine Analyse auf der Meso-Ebene sozialer und symbolischer Bindungen, mit Verweisen auf die Mikro-Ebene der Präferenzen von Migranten und auf die Makro-Ebene struktureller Bedingungen wie Einkommensunterschieden und Differenzen im Hinblick auf politische Freiheit zwischen Ländern.

(2) Kettenmigration in Migrationssystemen bei relativ hohen Freiheitsgraden

Unter der Voraussetzung, dass für potenzielle Migranten der Spielraum für Entscheidungen relativ hoch ist, entwickelt sich transnationale Migration innerhalb starker Beziehungen zwischen Emigrations- und Immigrationsländern in den erwähnten Migrationssystemen. Die relativ hohe Entscheidungsfreiheit als weitgehende Abwesenheit äußerer Zwänge zur Flucht trifft dabei nicht auf alle potenzielle Migranten zu, sondern nur auf diejenigen, welche die Entscheidungen fällen, z.B. die Familienoberhäupter. Um ursprünglich ortsgebundene Ressourcen zu mobilisieren, bedarf es Transmissionsriemen wie grenzüberschreitenden sozialen und symbolischen Bindungen, die vorzugsweise von Pioniermigranten etabliert werden. Man sollte dabei nicht vorschnell von der Bedeutung von Migranten- bzw. Migrationsnetzwerken sprechen, sondern immer angeben, in welchen sozialen Formationen - z.B. Familie, Dorfgemeinschaften, Freundeskreise und Cliquen - und mit Hilfe welcher Ressourcen in sozialen und symbolischen Bindungen sich transnationale Migration vollzieht.

Falls über soziale und symbolische Bindungen in bereits existierenden grenzüberschreitenden Gruppen und Organisationen die Mechanismen der Reziprozität und Solidarität in Familien, Nachbarschaften, Dorf- und anderen Gemeinschaften leicht in Anspruch genommen werden können, sind die Aussichten für Ketten- und Massenmigration aus wenigen Regionen des Südens in wenige des Nordens besonders gut. Mit anderen Worten: Wenn sich solche selbst reproduzierenden Dynamiken der Migration entwickeln können, kommt es zu relativ freiwilliger Kettenmigration.

${ }^{8}$ Eine sehr weit die Synthese unterschiedlicher Analyseebenen vorantreibende Typologie stammt von Shmuel Eisenstadt (1954: 3) in Anlehnung an Talcott Parsons (1951). Allerdings zielt der Eisenstadt'sche Vorschlag schon auf die Integration von Immigranten. 
Sind die Freiheitsgrade potenzieller Migranten dagegen sehr gering, wie üblicherweise in Fällen erzwungener Migration in Folge von zwischenstaatlichen Konflikten, sozialen Revolutionen, Bürgerkriegen und Umweltzerstörung, ist es unwahrscheinlich, dass sich selbst reproduzierende Migrationsdynamiken entstehen. Und wenn sie vorkommen, dann sind sie oft nur von sehr kurzer Dauer oder verlaufen nur über kurze Distanz. Ein Beispiel für das letztgenannte Phänomen sind etwa guatemaltekische Flüchtlinge in Mexiko in den 1980er Jahren. Allerdings folgen Flüchtlinge manchmal durchaus etablierten Pfaden der Arbeitsmigration. Als Beispiel ließen sich manche Asylbewerber aus der Türkei anführen, die Ende der 1970er und während der 1980er Jahre als Kurden in Ländern wie Deutschland und den Niederlanden Asyl ersuchten, während ähnliche Kategorien in den 1960er Jahren als sog. Gastarbeiter wanderten. Dabei ist darauf hinzuweisen, dass die rechtlichen Kategorien „Arbeitsmigrant“ und „Asylbwerber“ nicht viel über die Ursachen von Migration aussagen.

Die Frage ist nun, wie weit gerade bei Kettenmigration mit relativ hohen Freiheitsgraden von Migranten die sich selbst verstärkenden bzw. endogenen Dynamiken verlaufen und ob und wie diese unterbrochen werden. Generell lässt sich bei relativ freiwilliger Migration die Entwicklung und Diffusion von Migrationsprozessen stilisiert in Gestalt einer S-Kurve abbilden. Eine solche allgemeine soziale Diffusionskurve, die auch im spezifischen Fall der Migration vorkommt, ergibt sich, wenn man die Anzahl jeweiliger Migranten (y-Achse) auf einer Zeitachse (x-Achse) kumulativ anordnet (Schaubild 1) ${ }^{9}$ : Drei Phasen lassen sich in idealtypischer Weise unterscheiden (grundlegend zu sozialen Diffusionsprozessen, siehe Rogers 1983: 243-5).

Phase 1 - Initialphase. Der Anstieg der Kurve ist noch relativ flach. Pioniermigranten legen auf der Grundlage von Anwerbungen formaler oder informeller Art einen Grundstock. Die Selektion potenzieller Migranten kommt hauptsächlich in drei Formen vor: Anwerbung durch Organisationen, persönlicher Kontakt und indirekte („strukturelle“) oder brutale Gewalt. In allen drei Situationen handeln Migranten in der Regel nicht isoliert voneinander. Die Mechanismen Reziprozität und Solidarität sind in allen drei Kontexten operativ. Bei formaler Anwerbung erhalten Pioniermigranten eine Chance, ins Ausland zu wandern. Als Beispiel sei an die so genannte Gastarbeiteranwerbung der 1960er Jahre erinnert. Später offerieren Pioniermigranten teilweise wiederum Ressourcen für den Aufbau netzwerkartiger Strukturen

${ }^{9}$ Die stilisiert auf Migrationsprozesse anwendbare allgemeine Diffusionskurve ist in ihrem Verlauf identisch mit der umgekehrten U-Kurve, wenn man die Daten nicht kumulativ, sondern zu einem bestimmten Zeitpunkt abbildet und die x-Achse „Zeit“ durch „BSP pro Kopf“ ersetzt. Allerdings bezeichnet die S-Kurve einen dynamischen Zusammenhang kumulativer Kausalität, während die umgekehrte U-Kurve jeweils nur Momentaufnahmen einer statistischen Korrelation abbildet. 
auf Gemeinde-, Nachbarschafts- oder Verwandtschaftsebenen. Die zweite Form, die der persönlichen Kontakte, charakterisiert die überwältigende Mehrheit der Bindungen nach der Initialphase. Der dritte Typ, direkte Gewalt, weist darauf hin, dass strukturelle Einschränkungen darauf einwirken, welche Strategien potenzielle Migranten überhaupt verfolgen können.

Diese Formen der Mobilisierung sorgen prinzipiell für die Transferierbarkeit von kulturellem, sozialem und symbolischem Kapital. In dieser ersten Phase werden die Grundlagen für die Mobilität von zunächst lokal gebundenem Kapital gelegt, indem Ressourcen in sozialen und symbolischen Bindungen aktiviert werden. Makler mit Bindungen zu verschiedenen Bezugsgruppen spielen dabei eine zentrale Rolle, indem sie als „lachende Dritte“ (tertius gaudens) Nutzen aus verschiedenartigen Verflechtungen ziehen und dabei zur Bildung einer kritischen Masse an Pioniermigranten beitragen (Simmel 1992: Teil VI). Ganz wichtig ist hierbei die Einsicht, dass spezifische Reziprozität, die nur auf direkt gegenseitigen Beziehungen beruht und fokussierte Solidarität, die nur auf spezifische Gruppen wie Verwandtschaftssysteme zielt, nicht ausreichen, um Massenmigration in Gang zu bringen. Spezifische Reziprozität und fokussierte Solidarität ermöglichen nur Wanderung in relativ engen Familienverbänden und diffundieren in der Regel nicht darüber hinaus. Für Kettenmigration sind Formen generalisierter Reziprozität und diffuser Solidarität Voraussetzung, d.h. die Mechanismen schließen über Bindungen von Maklern auch „Dritte“ außerhalb der engeren Bezugsgruppen mit ein. Andernfalls stagniert Migration relativ schnell (siehe dazu die in diesem Sinne interpretierbare Fallstudie von Engelbrektsson 1978).

Phase 2 - Beschleunigungsphase: Auf der Grundlage der Mobilisierung von Mechanismen wie generalisierter Reziprozität und diffuser Solidarität steigt die Zahl der Migranten steil, zumeist sogar exponentiell an. Weiterhin werden dadurch die bereits bestehenden Bindungen zwischen Emigrations- und Immigrationsländern und den dazugehörigen Regionen verstärkt. Jeder Fall von Migration tendiert wie bei Prozessen der Immobilität dann dazu, einen sich selbst verstärkenden Effekt zu erzeugen, indem die Ressourcen den Trend beschleunigen. Im Falle der Beschleunigung beteiligen sich immer mehr potenzielle Migranten in immer kürzerer Zeit an Wanderungen. Diese sind in der Regel eingebettet in soziale und symbolische Bindungen mit den entsprechenden Ressourcen. Wir können diese Beobachtungen also wiederum nach dem Prinzip der kumulativen Kausalität zusammenfügen. Damit werden auch die externen Ursachen, die transnationale Migration überhaupt erst ermöglichen, wie beispielsweise offizielle Anwerbung, im Zeitverlauf proportional weniger relevant für den weiteren Verlauf. Die Bedeutung der Eigendynamik nimmt hingegen zu. Eine Folge davon ist, dass bei vorhandenen - darunter auch „schwachen“ Bindungen - Migration immer weniger sozial selektiv verläuft. So werden 
allmählich auch vermehrt soziale Klassen unterhalb häufig in eine als „Mittelklasse“ zu bezeichnenden Kategorie mit einbezogen (Massey et al. 1987). Das exponentielle Wachstum trägt zum beschleunigten, grenzübergreifenden Transfer ganz verschiedener Kapitalsorten bei. Gerade im Falle der Arbeitsmigration von Personen, die in wenig qualifizierten Tätigkeiten in Immigrationsländern Beschäftigung finden, wird die endogene Dynamik nicht zuletzt dadurch verstärkt, dass manche Kapitalsorten konvertibel sind. Soziales Kapital vermag beispielsweise bei Wohnungssuche oder Kindererziehung finanzielles Kapital substituieren, indem Migranten aus der gleichen Region einander behilflich sind.

Oft kommt es gerade in dieser zweiten Phase zu illegaler bzw. irregulärer Migration. Dabei könnte man von einem "Tocqueville-Effekt" sprechen. Bekanntlich hat schon Alexis de Tocqueville in den 1830er Jahren auf das Phänomen verwiesen (Tocqueville 1951), das wir heute als relative Deprivation bezeichnen, also die Beobachtung, dass kollektives Handeln ein Resultat eines Prozesses sein kann, indem die Beteiligten gerade auf der Grundlage von Verbesserungen höhere Ansprüche stellen. Die subjektiven Erwartungen steigen dann schneller als die objektiven Gelegenheiten. Für den vorliegenden Zusammenhang lässt sich die Theorie rationalen Handelns mit der These „relativer Frustration“ verbinden (locus classicus: Stouffer 1949). Das Beispiel, das Pioniermigranten für die Zurückgebliebenen setzen, führt zu einem sich selbst verstärkenden Prozess, in dem die Motivationen einzelner Personen im Vergleich zur kollektiven Erwartung der Abwanderung immer weniger wichtig werden. Dies bedeutet auch, dass die Wünsche bzw. Präferenzen der potenziellen Migranten im Gefolge der wahrgenommenen Reduktion von Transport-, Transfer- und Transaktionskosten schneller wachsen als die real vorfindbaren Möglichkeiten zur grenzüberschreitenden Abwanderung. Zentral gründen endogene und sich selbst verstärkende Prozesse nicht einfach auf abnehmenden Informationskosten durch Migrationsnetzwerke - wie etwa einfache Theorien rationalen Handelns voraussagen würden - sondern auch auf der Transformation der Präferenzen von Sesshaften, die unter anderem negative Rückkopplungseffekte wie abnehmenden sozialer Status aufgrund von NichtMigration fühlen. Klafft die Schere von Präferenzen und Gelegenheiten immer weiter auseinander, so kommt es aufgrund hoher Frustration angesichts fehlender Möglichkeiten zu legalen Wegen der Migration häufig zu einem Anwachsen der Wanderung über staatlich nicht autorisierte Kanäle - was etwa im Falle Mexiko-USA seit den 1970er Jahren vorzüglich anschaulich wird (Mackie 1995). Empirisch hilft diese Perspektive beispielsweise die Beobachtung zu erklären, dass es nach längerer offizieller Anwerbung zu verstärkter „illegaler“ Migration kommt. Das trifft nicht nur auf den mexikanisch-amerikanischen Fall in den 1990er Jahren zu, sondern auch auf die türkisch-deutsche Migration Ende der 1960er Jahre. Die provokative Schlussfolgerung des Modells relativer Frustration wäre, dass 
geschlossene oder offene Grenzen auf Seiten der Immigrationsländer keine GelegenheitsPräferenz-Spiralen in Gang setzen. Demgegenüber ist dies bei partiellen Grenzöffnungen, also bei der Mehrzahl der tatsächlich vorfindbaren Migrationspolitiken auf der Empfängerseite, durchaus der Fall. Dabei ist allerdings zu beachten, dass sich die Präferenzänderungen bei vielen Migranten sich nur dann in tatsächliche Migration umsetzen lassen, wenn neben der Transferierbarkeit kulturellen Kapitals gerade bei nicht privilegierten Arbeitsmigranten Ressourcen wie generalisierte Reziprozität und diffuse Solidarität mobilisiert werden können, die bei der Suche nach Wohnung, Arbeitsplatz und Kinderbetreuung zum Tragen kommen.

\section{Phase 3 - Konsolidierung, Remigration und mögliche transnationale Verdichtung: Es} folgt ein annäherungsweise linearer Anstieg, der aber immer weiter abflacht - bis er schließlich kaum noch wahrnehmbar ist. An diesem letzten Punkt erschöpft sich das jeweilige Reservoir potenzieller transnationaler Migranten: Alle Personen einer bestimmten Region, die zu migrieren beabsichtigten, haben mittlerweile ihren Plan in die Tat umgesetzt. Es migrieren dann nicht nur die erwerbswirtschaftlich Tätigen, sondern auch vermehrt Kinder und Ältere; nicht nur die Mitteklasse, sondern auch ärmere Einkommenskategorien (Massey et al. 1994).

Im Verlauf von Migrationsprozessen entwickeln sich in der Regel auch Remigrationsverläufe. Die empirische Forschung über Migration stimmt darüber überein, dass die Remigrationsquote bei hohen Freiheitsgraden eine signifikante Minderheit umfasst (zusammenfassend: Richmond 1984). Es kommt bei kettenartigen Migrationsprozessen aus wenigen Orten des Südens in ausgewählte Länder des Nordens und dabei wiederum in relativ wenige Regionen innerhalb dieser Staaten zu räumlichen Clusterbildungen. Neben den insbesondere bei relativ gering qualifizierten Arbeitsmigranten und deren Angehörigen beobachtbaren Konzentrationen in „ethnischen Kolonien“ und Arbeitsmärkten wie z.B. beruflichen Nischen lassen sich auch bei relativ hochqualifizierten Migranten und unternehmerisch orientierten Migranten sektorale Clusterbildungen feststellen. Dies trifft nicht nur auf Süd-Nord-Wanderungen, sondern auch auf Nord-Süd-Remigration zu. Ein Beispiel für Clusterbildung sind chinesische Computerspezialisten in Kaliforniens Silicon Valley, die in den letzten zwanzig Jahren durch Unternehmensgründungen fast ein Fünftel aller Unternehmen und einen noch höheren Anteil aller neuen Arbeitsplätze schufen. Auch bei Remigration entstehen ähnliche Effekte: So sind Rückkehrmigration von Spezialisten und Unternehmensgründungen in Taiwan, etwa im Hsinchu-Science-based Industrial Park aufzeigbar (Saxenian 1999). Wichtig ist nun, dass in diesem und in ähnlich gelagerten Fällen Rückkehrmigration nach ähnlichen kumulativen Kausalitäten verlief wie die primäre Emigration. Neben makrostrukturellen Faktoren wie die Marktöffnung in den ursprünglichen 
Herkunftsländern spielt gerade auch diffuse Solidarität unter Migranten und dazugehörige kulturelle Deutungsmuster eine wichtige Rolle in der endogenen Verstärkung von Remigrationsprozessen. Für indische Rückkehrmigranten aus den USA und Großbritannien spielt beispielsweise eine wichtige Rolle, dass Kinder in einem speziellen kulturellen Umfeld aufwachsen und dass Ehen zu arrangieren sind (Khadria 1999). Je stärker nun die wirtschaftlichen Erfolge und die soziokulturelle Integration von Remigranten sichtbar sind, desto größer der Anreiz für andere Immigranten, diesem Beispiel zu folgen. Aus den vorliegenden konzeptuellen Annahmen und empirischen Befunden wird für die in den 1960er Jahren schon aktuelle und ab den 1990er Jahren wieder aufgekommene Debatte um die Wirkungen der Migration von hochqualifizierten Spezialisten auf die Ökonomien der Immigrations- und Emigrationsländer schnell deutlich: Die in den Gegensatzpaaren von „brain drain“ als Humankapitalverlust für die Sendeländer und von „brain gain“ als Gewinn angenommene Wirkung (z.B. Barré et al. 2003) kann nur dann empirisch solide erfasst werden, wenn das Phänomen der „brain circulation“ ausreichend berücksichtigt wird, d.h. die außerordentlich hohe Bereitschaft von Migranten zu Rückkehrmigration, Weiterwanderung und bei Niederlassung zu dichten, kontinuierlichen Bindungen über Staatsgrenzen hinweg. Es wäre dabei verkürzt, Austauschprozesse von Wissen und kulturellem Kapital auf die physische Mobilität von Personen zu reduzieren. In etablierten Migrationssystemen existieren Verbünde von Wissenschaftlern, teilweise gar „epistemische Gemeinschaften“ (Haas 1992) mit gemeinsamen Grundüberzeugungen und professionellen Standards, die über weit entfernte geographische Räume hinweg eng kooperieren. Eine Studie der USamerikanischen National Science Foundation deutet darauf hin, dass gerade Wissenschaftler, die sich etwa in den USA auf Dauer niedergelassen haben, besonders häufig und intensiv mit Kollegen aus ihren Herkunftsstaaten kooperieren - ob diese nun in OECD- oder Entwicklungsländern wohnen (Regets 1999).

Trotz kumulativer Effekte erreichen Wanderungsströme Wendepunkte, ab denen transnationale Migration nicht mehr weiter wächst. Alle potenziellen Migranten aus den wenigen Orten haben dann ihr Wanderungsziel verwirklicht. Mittel- und langfristig gesehen vermischen sich allerdings wiederum endogene mit exogenen Faktoren, da Immigrationsstaaten als nationale Wohlfahrtsstaaten zwar häufig an „billigen und willigen“ bzw. hoch spezialisierten Fachkräften interessiert sind, aber keine offenen Grenzen dulden. Die meisten der Süd-NordWanderungen haben daher gegenwärtig ihre sozial-endogen erzeugten Wendepunkte in Phase 3 aus innenpolitischen Gründen der OECD-Länder nicht erreicht. Dies kann u.a. am Fall türkisch-deutscher Migration illustriert werden (Schaubild 2). In Bezug auf bestimmte Kategorien von Immigranten haben restriktive politische Maßnahmen der Immigrationsstaaten als Reaktion auf die interne Globalisierung wie etwa infrastrukturelle 
Engpässe, vermutete Arbeitsmarktkonkurrenz und unerwünschte kulturelle Pluralisierung sich selbst verstärkende Migrationsprozesse signifikant beschränkt. Im vorliegenden Falle war es bekanntlich der Anwerbestopp von 1973, wenn auch aufgrund humanitärer, völkerrechtlicher Verpflichtungen der Familiennachzug ${ }^{10}$ nicht zum Erliegen kam und später Fluchtmigration folgte. Nicht ausgereizte S-Kurven sind also im Wesentlichen auf die Politik und Politiken der Empfängerländer zurückzuführen, die asymmetrisch sowohl Prozesse transnationaler Migration bei relativ hoher Autonomie von Migranten ins Rollen bringen, aber auch einseitig Anwerbeprozesse zu stoppen vermögen.

Über Remigration hinaus lautet die Frage, ob beim Auslaufen der S-Kurve wiederum Migrationsmuster entstehen, welche Migrationsverhalten perpetuieren, so genannte Kulturen der Migration. Dies bedeutet, dass transnationale Migration, insbesondere in bestimmten Lebensphasen, über Diffusion hinaus als ein akzeptiertes Muster der räumlichen und sozialen Mobilität angesehen wird. Kulturen der Migration umfassen oft mehrere Generationen, wie es etwa auf manchen Inseln der Karibik als Phase in Lebensläufen deutlich wird (u.a. Pessar 1997). Derartige Muster sind ein wichtiger Hinweis darauf, dass nicht nur Integration im Immigrationsland oder Rückkehrmigration ins Herkunftsland Migrationsverläufe bestimmen, sondern auch Zirkulation zwischen den Emigrations- und Immigrationsregionen. Kulturen der Migration wären somit ein Typ eines Phänomens, das mit vielfältigen Begriffen wie Transnationalismus, transnationale Gemeinschaft, transnationale Kreisläufe, transnationaler bzw. transstaatlicher sozialer Raum (Faist 2000b), transnationale Formation, transnationales soziales Feld (Basch et al. 1994) bzw. ein Teil von „offenen Räumen“ (Münch 2001) bezeichnet wird.

Allerdings ist zu beachten, dass derartige Transnationale Soziale Räume nicht einfach auf physischer Mobilität beruhen. Soziale und symbolische Bindungen basieren nicht nur oder gar primär auf geographischer Nähe. Neue Technologien wie Telefon und Flugverkehr ähnlich der Welle im 19. Jahrhundert mit Telegraph und Dampfschiff - ermöglichen heute einen kontinuierlichen Austausch auch über Reisen hinaus. Alle transnationalen Konzepte verweisen auf plurilokale Bindungen von Personen, Cliquen, Gruppen und Organisationen, die in mehreren Orten über die jeweiligen Staatsgrenzen hinweg relativ kontinuierlich und dicht miteinander verbunden sind. Dabei entstehen teilweise Kreisläufe von Menschen,

${ }^{10}$ Dabei handelt es sich beispielsweise in Deutschland seit den 1980er Jahren um relativ geringe absolute Zahlen und Quoten. Gerade einmal etwa 12.000 Kinder unter 18 Jahren von Immigranten aus der Türkei und ex-Jugoslawien - zusammen machen sie über die Hälfte aller Immigranten in der Bundesrepublik aus - hatten im Jahre 2001 ihren Wohnsitz in diesen beiden Ländern und kämen für einen Nachzug in Betracht (Meier-Braun 2002: 51-2). 
Waren, Geld, Symbolen, Ideen und kulturellen Praktiken, die über die Zeit der Wanderungsprozesse selbst die Sozialintegration von Immigranten in nationalstaatlich verfassten Gesellschaften berühren. Transnationale Soziale Räume sind also nicht nur Produkte kumulativer Kausalität, sondern perpetuieren sich wiederum selbst. Sie können über mehrere Generationen hinweg existieren, nicht nur in den Kulturen von Migration, sondern auch als Diaspora, etwa die fünften und sechsten Generationen von jüdischen oder polnischen Einwanderern in den USA, die Bewegungen in ihren Heimatländern unterstützen.

Transnationale Soziale Räume sind allerdings keine quasi-automatischen Produkte transnationaler Migration. Es gibt Migrantengruppen, die schon bei ihrer Ankunft im Aufnahmeland überwiegend den Kontakt in das Herkunftsland abbrechen. Dazu zählen etwa (Spät-)Aussiedler aus der ehemaligen UdSSR in Deutschland. Zweitens ist für andere Gruppen Transnationalität ein charakteristisches Merkmal ihres Wanderungs- und Integrationsverhalten, das nicht erst im Migrationsverlauf kumulativ entsteht. Dazu gehören Migrantenkategorien, die kaum Beziehungen in das Herkunftsland pflegen, dafür aber Kontakte in Ländern der geplanten Weitermigration erneuern und/oder entwickeln. Jüdische Immigranten aus Russland sind ein Beispiel dafür. Sie pflegen nach der Ankunft in Deutschland vorwiegend Kontakte nach Israel oder in die USA (Gould 2002).

\section{Transnationale Migration: Transnationalisierung und Universalisierung}

Transnationale Migration passt insgesamt nicht einfach in das Schema zunehmender Häufigkeit und Dichte von grenzübergreifenden Strömen - wie das etwa in gängigen Globalisierungsinterpretationen im Sinne von Transnationalisierung zu erwarten wäre. Und auch der Hinweis darauf, dass die Globalisierung massenmedialer Kommunikation, Telekommunikation und Transport oder die Universalisierung von Menschenrechten und Demokratie und der damit verbundenen Ausreisefreiheit transnationale Migration fördere, wirft kaum Licht auf die eigentümliche Konstellation relativer transnationaler Immobilität.

Schon die Frage danach, ob es sich angesichts der Zahl von Migranten bzw. des Anteils transnationaler Migranten an der Weltbevölkerung um einen Fall von Globalisierung oder Nicht-Globalisierung handele, greift zu kurz. Ein Blick auf die Ursachen räumlicher Mobilität über Staatsgrenzen hinweg ergibt, dass vielfältige Restriktionen für Süd-Nord-Migration existieren. Dies fängt bei orts-, regional und landesspezifischen Ressourcen von Migranten an, die nicht beliebig über Grenzen transferierbar sind und reicht bis hin zu protektionistischen Politiken von Wohlfahrtsstaaten im Norden. Dazu kommen zusätzliche Faktoren, wie zum 
Beispiel die weitgehende Abwesenheit internationaler Regimebildung im Bereich der Arbeitsmigration (Miller 1992) und die mangelnde Durchsetzungsfähigkeit internationaler Vereinbarungen zum Schutz von Migranten, was etwa im Falle der Konventionen der Internationalen Arbeitsorganisation (ILO) auffällig ist (Böhning und Werquin 1989). Derartige Phänomene lassen sich schwerlich in die Schablone eines trotz Unterbrechungen weitgehend linearen Wachstums grenzübergreifender Flüsse einordnen. Bei all den Gemeinsamkeiten, die grenzübergreifende Flüsse von Personen mit denen von Kapital und Gütern aufweisen, ist mindestens ein ganz zentraler Unterschied zu beachten: Ein Großteil der Ressourcen transnationaler Migranten ist lokal spezifisch. Dabei verändern die Prozesse der kumulativen Immobilität selbst wiederum den Gebrauch der vorhandenen sozialen und symbolischen Bindungen. Dies geschieht dergestalt, dass durch sich selbstverstärkende Effekte Transaktionskosten für lokale Bindungen verringert werden und somit Immobilität über in situ Adaptation gefördert wird. Im umgekehrten Falle reduzieren Transmissionsriemen wie Anwerbung und Makler die Transaktionskosten für grenzübergreifende Bindungen und befördern damit Ketten- und Massenmigration aus einigen wenigen Orten. Summa summarum ist in Prozessen transnationaler Migration neben der Transnationalisierung von Personenwanderungen, der Universalisierung von Rechten und der internen Globalisierung als protektionistische Reaktion auf transnationale Migration gerade die Transnationalisierung lebensweltlicher Bezüge von Migranten ein entscheidendes Merkmal dessen, was gemeinhin Globalisierung genannt wird. Nur in der Dualität von Ortsgebundenheit und Mobilität von Ressourcen potenzieller und tatsächlicher Migranten kann das Rätsel der relativen Immobilität in einer globalisierten Welt hinreichend beschrieben und analytisch erfasst werden.

Über die Evaluation der Wirkung transnationaler Migration hinaus leistet die Beschreibung und Erklärung des Rätsels der relativen Immobilität in der transnationalen Migration auch einen konzeptuellen Beitrag zur Erfassung transnationaler Bindungen. Der weitgehend diffuse Begriff Globalisierung bezieht sich auf Prozesse, welche die ganze Welt umspannen und als „action at distance - the increasing interpenetration between individual lives and global futures“ (Giddens 1991: 26) gesehen werden kann. Eine solche Auffassung weist darauf hin, dass sich neben wirtschaftlichen Austauschprozessen auch soziale und symbolische Beziehungen sowie die darin inhärenten Ressourcen wie Reziprozität und Solidarität räumlich immer mehr ausdehnen. Einige Globalisierungstheoretiker des 19. Jahrhunderts gingen schon so weit, die voranschreitende Zerstörung von Raum-Zeit durch den Verwertungsprozess des ökonomischen Kapitals zu konstatieren (Marx 1974: 438). Die für den vorliegenden Zusammenhang interessantesten Vertreter situieren sich heutzutage zwischen Hyper-Globalisierern und Globalisierungsskeptikern. Auf der einen Seite extrapolieren die Hyper-Globalisierer die Zukunft aus den Gesetzen des Marktes heraus, und manche wenden diese Logik auf alle 
Sphären an: Alles ende in einer zunehmenden universellen Verdichtung von Zeit und Raum. Dazu zählen auch die Vertreter der These der Deterritorialisierung, die ein allgemeines Anwachsen von Hypermobilität im Anzug sehen (Waldron 1992). Migranten gerinnen dann zu Prototypen eines kosmopolitischen Lebensstils. Auf der anderen Seite dieses intellektuellen Globus verweisen die Globalisierungsskeptiker darauf, dass in vielerlei Hinsicht die Weltökonomie Ende des 19. Jahrhunderts noch integrierter war als heute. Sie folgern daraus, dass Globalisierung nichts Neues sei und dass es keine fundamentale Umstrukturierung des internationalen Systems gäbe. Wohl aber ließen sich Perioden verstärkter und beschleunigter Vernetzung beobachten (Hirst und Thompson 1996). Weiterführend ist hier die Idee einer Vorstellung zwischen „space of places“ (Ruggie 1998) und „space of flows“ (Harvey 1989). Dieser Gedanke bezeichnet den konzeptuellen Raum zwischen einem ausschließlichen Fokus auf grenzenlosen Tauschprozessen und der Verflüssigung von Raum und Zeit in einer Netzwerkgesellschaft (vgl. Castells 1996) einerseits und einer Konzentration auf die Interaktion container-räumlicher Einheiten wie Staaten andererseits. 


\section{Literatur}

Appleyard, Reginald, 1992: Migration and Development: A Critical Relationship, Asian and Pacific Migration Journal 1, 1: 1-19.

Bade, Klaus J. und Rainer Münz, 2002: Migrationsreport 2000. Fakten - Analysen - Perspektiven. Frankfurt a. M./ New York: Campus Verlag.

Barré, Philippe, Victor Hernandez, Jean-Baptiste Meyer, Dominique Vinck (Hrsg.), 2003:

Diasporas scientifiques. Expertise collégiale. Institut de Recherche sur le Développement. Paris: Ministère des Affaires Etrangères.

Basch, Linda, Nina Glick Schiller and Cristina Szanton Blanc, 1994: Nations Unbound: Transnational Projects, Postcolonial Predicaments, and Deterritorialized Nation-States. Langhorne, PA: Gordon and Breach.

Bilsborrow, Richard, Graeme Hugo, A. S. Oberai und Hania Zlotnik, 1997: International Migration Statistics: Guidelines for Improving Data Collection Systems. Geneva: International Labour Office (ILO).

Birg, Herwig, 2001: Die demographische Zeitenwende. Der Bevölkerungsrückgang in Deutschland und Europa. München: Verlag C.H. Beck.

Böhning, Wolf-Rüdiger und Jacques Werquin, 1989: Some Economic, Social and Human Rights Considerations Concerning the Future Status of Third-Country Nationals in the Single European Market. Geneva: International Labour Office (ILO).

Bourdieu, Pierre, 1983: Ökonomisches Kapital, kulturelles Kapital, soziales Kapital, in Reinhard Kreckel (Hrsg.), Soziale Ungleichheiten (Soziale Welt, Sonderheft 2). Göttingen: Otto Schwartz \& Co.: 183-198.

Castells, Manuel, 1996: The Rise of Network Society. Oxford: Blackwell.

Castles, Stephen and Mark J. Miller, 1993: The Age of Migration: International Population Movements in the Modern World. London: Macmillan.

Coleman, James S., 1990: Foundations of Social Theory, Cambridge, MA: The Belknap Press of Harvard University Press. 
Dowty, Alan, 1987: Closed Borders: The Contemporary Assault on Freedom of Movement. New Haven: Yale University Press.

Durkheim, Émile, 1965 [1898]: Individual and Collective Representations, in ders.: Sociology and Philosophy, New York: The Free Press, 1-34.

Eisenstadt, Shmuel N., 1954: The Absorption of Immigrants. A Comparative Study Based Mainly on the Jewish Community in Palestine and the State of Israel. London: Routledge.

Engelbrektsson, Ulla-Britt, 1978: The Force of Tradition: Turkish Migrants at Home and Abroad. Göteborg: Acta Universitatis Gothoburgensis.

Esser, Hartmut, 1980: Aspekte der Wanderungssoziologie. Assimilation und Integration von Wanderern, ethnischen Gruppen und Minderheiten. Darmstadt und Neuwied: Luchterhand.

Eurostat, 1995: Causes of International Migration. Luxemburg: Office for Official Publications of the European Communities.

Eurostat, 2000: Push and Pull Factors of International Migration: A Comparative Report. Luxemburg: Office for Official Publications of the European Communities.

Faini, Riccardo und Alessandra Venturini, 1994: Migration and Growth: the Experience of Southern Europe. Discussion Paper Series No. 964. London: Centre for Economic Policy Research.

Faist, Thomas, 2000a: The Volume and Dynamics of International Migration and Transnational Social Spaces. Oxford: Oxford University Press.

Faist, 2000b: Transstaatliche Räume. Wirtschaft, Politik und Kultur in und zwischen Deutschland und der Türkei. Bielefeld: transcript.

Faist, Thomas, 2004: Towards a Political Sociology of Transnationalization, European Journal of Sociology 45, $3: 19-54$.

Faist, Thomas und Andreas Ette (Hrsg.), 2007: Between Autonomy and the European Union: The Europeanization of National Immigration Policies. Houndmills, UK: Palgrave Macmillan.

Findlay, Alan, 2003: Skilled Transients: The Invisible Phenomenon? Robin Cohen (Hrsg.), The Cambridge Survey of World Migration. Cambridge: Cambridge University Press, 515523.

Giddens, Anthony, 1991: The Consequences of Modernity. Cambridge: Polity Press. 
Gold, Steven J., 2002: The Israeli Diaspora. Seattle: University of Washington Press.

Gouldner, Alvin W., 1960: The Norm of Reciprocity: A Preliminary Statement, American Sociological Review 25, 1: 161-78.

Granovetter, Mark S., 1979: The theory gap in social network analysis, in Paul W. Hollandund Stephen Leinhardt (eds.), Perspectives in Social Network Research, New York: Academic Press, 45-67.

Haas, Peter M., 1992: Introduction: Epistemic Communities and International Policy Coordination, International Organization 46 (Sonderheft): 1-35.

Hägerstrand, Torsten, 1975: On the Definition of Migration, in Emrys Jones (ed.), Readings in Social Geography. Oxford: Oxford University Press, 200-10.

Hammar, Tomas et al. (eds.), 1997: International Migration, Immobility and Development: Multidisciplinary Perspectives. Oxford: Berg.

Harvey, David, 1989: The Condition of Postmodernity. Oxford: Blackwell.

Held, David, Anthony McGrew, David Goldblatt und Jonathan Perraton, 1999: Global Transformations: Politics, Economics and Culture. Stanford, CA: Stanford University Press.

Hirschman, Albert O., 1970: Exit, Voice, and Loyalty: Responses to Decline in Firms, Organizations, and States. Cambridge, MA: Harvard University Press.

Hirst, Paul und Grahame Thompson, 1996: Globalization in Question: The International Economy and the Possibilities of Governance. Cambridge: Polity Press.

Hollifield, James, 1992: Immigrants, Markets and States: The Political Economy of Postwar Europe. Cambridge, MA: Cambridge University Press.

Hugo, Graeme J., 1981: Village-Community Ties, Village Norms and Ethnic and Social Networks: A Review of Evidence from the Third World, in Gordon F. De Jong und Richard W. Gardner (eds.), Migration Decision Making: Multidisciplinary Approaches to Microlevel Studies in Developed and Developing Countries. New York: Pergamon Press, 186-224.

Huntington, Samuel P., 1991: The Third Wave: Democratization in the late Twentieth Century. Norman: University of Oklahoma Press.

Khadria, Binod, 1999: The Migration of Knowledge Workers: Second-Generation Effects of India's Brain Drain. New Delhi: Sage Publications. 
Kibria, Nazli, 1993: Family Tightrope: The Changing Lives of Vietnamese Americans. Princeton, $\mathrm{NJ}$ : Princeton University Press.

Kritz, Mary M., Lin Lean Lim und Hania Zlotnik (eds.), 1992: International Migration Systems: A Global Approach. Oxford: Clarendon Press.

Kunz, Egon F., 1973: The Refugee in Flight: Kinetic Models and Forms of Displacement, International Migration Review 7, 2: 125-146.

Kuptsch, Christiane and Peng Eng Fong (eds.), 2005: Competing for Global Talent. Geneva: International Labour Office (ILO) and Singapore: Wee Kim Wee Centre.

Kyle, David, 2000: Transnational Peasants: Migrations, Networks, and Ethnicity in Andean Ecuador. Baltimore, MD: Johns Hopkins University Press.

Lee, Everett S., 1964: A Theory of Migration, Demography 3: 47-57.

Lerner, Daniel, 1958: The Passing of Traditional Society, Glencoe, IL: The Free Press.

Mackie, Gerry, 1995: Frustration and Preference Change in Immigration Migration, Archives Européennes de Sociologie 36, 2: 185-208.

Massey, Douglas S., Rafael Alarcón, Jorge Durand und Humberto González, 1987: Return to Aztlán: The Social Process of International Migration from Western Mexico. Berkeley, A: University of California Press.

Massey, Douglas S., Jorge Arango, Graeme Hugo, Ada Kouaouci, Antonio Pellegrino und Edward Taylor, 1993: Theories of International Migration: A Review and Appraisal, Population and Development Review 19, 3: 431-466.

Massey, Douglas S., Luin Goldring, Jorge Durand, 1994. Continuities in Transnational Migration: an Analysis of Nineteen Mexican Communities, American Journal of Sociology 99 (May): 1492-533.

Maruyama, Magoroh, 1963: The Second Cybernetics: Deviation-Amplifying Mutual Causal Processes, American Scientist 51, 2: 164-179.

McPhail, Clark und David Miller, 1973: The Assembling Process: A Theoretical and Empirical Examination, American Sociological Review 38, 6: 721-735.

Marx, Karl, 1974: Grundrisse der Kritik der Politischen Ökonomie. Berlin: Dietz Verlag. 
Meier-Braun, Karl-Heinz, 2002: Deutschland, Einwanderungsland. Frankfurt a.M.: Suhrkamp.

Miller, Mark J., 1992: Evaluation of Policy Modes for Regulating International Labour Migration, in Mary M. Kritz, Lin Lean Lim und Hania Zlotnik (eds.), International Migration Systems: A Global Approach. Oxford: Clarendon Press, 300-314.

Münch, Richard, 2001: Offene Räume. Soziale Integration diesseits und jenseits des Nationalstaats. Frankfurt a.M.: Suhrkamp.

OECD, 2000: Globalisation, Migration, and Development. Paris: Organisation for Economic Cooperation and Development.

Parsons, Talcott, 1951: The Social System. Glencoe, IL: The Free Press.

Pessar, Patricia R. (ed.), 1997: Caribbean Circuits: New Directions in the Study of Caribbean Migration. New York: Center for Migration Studies.

Portes, Alejandro (ed.), 1995: The Economic Sociology of Immigration: Essays on Networks, Ethnicity, and Entrepreneurship. New York: Russell Sage Foundation.

Putnam, Robert D., 1993: Making Democracy Work: Civic Traditions in Modern Italy. Princeton, NJ: Princeton University Press.

Regets, Mark C., 1999: Foreign Science \& Technology Personnel in the United States: An Overview of Available Data and Basic Characteristics, in OECD, Mobilizing Human Resources for Innovation. Paris: Organisation for Economic Co-Operation and Development.

Richmond, Anthony H., 1984: Explaining Return Migration, in Daniel Kubat (ed.), The Politics of Return: International Return Migration in Europe. Roma: Centro Studi Emigrazione \& New York: Center for Migration Studies, 269-76.

Rogers, Everett M., 1983: Diffusion of Innovations. Dritte Auflage. New York: The Free Press.

Ruggie, John Gerard, 1998: Constructing the World Polity: Essays on International Institutionalization. London: Routledge.

Saxenian, Anna L., 1999: Silicon Valley's New Immigrant Entrepreneurs. San Francisco, CA: Public Policy Institute of California.

Segal, Aron, 1993: An Atlas of International Migration. London: Hans Zell Publishers. 
Simmel, Georg, 1992 (1908): Soziologie. Untersuchungen über die Formen der Vergesellschaftung. Frankfurt: Suhrkamp.

Stouffer, Samuel A., 1949: The American Soldier. Princeton, NJ: Princeton University Press.

Strikewerda, Carl, 1997: Reinterpreting the History of European Integration: Business, Labor, and Social Citizenship in Twentieth-Century Europe, in Jytte Klausen und Louise A. Tilly (eds.), European Integration in Social and Historical Perspective. Lanham, MD: Rowman \& Littlefield, $51-70$

Thomas, William I. and Florian Znaniecki, 1927 [1918-1921]: The Polish Peasant in Europe and America, 5 Bände. New York: Alfred A. Knopf.

Tocqueville, Alexis de, 1951 [1835]: Über die Democratie in Amerika, Aus dem Französischen übertragen von Hans Zbinden. Zürich: Manesse.

Torpey, John, 2000: The Invention of the Passport: Surveillance, Citizenship and the State. Cambridge, MA: Cambridge University Press.

United Nations Secretariat, 2001: Replacement Migration: Is It a Solution to Declining and Aging Populations? Population Division, Department of Economic and Social Affairs. http://www.un.org/esa/population/publications/migration/migration.htm

United Nations Secretariat, 2005: Trends in Total Migrant Stock: The 2005 Revision Population Division, Department of Economic and Social Affairs. http://esa.un.org/migration

United Nations Development Programme (UNDP), 2006: Millennium Development Goals: A Compact Among Nations to End Poverty. Oxford: Oxford University Press.

United Nations High Commissioner for Refugees (UNHCR), 2006: The State of the World's Refugees: Human Displacement in the New Millennium. Oxford: Oxford University Press.

Waldron, Jeremy, 1992: Minority Cultures and the Cosmopolitan Alternative, University of Michigan Journal of Law Review 25, 3: 751-793.

Weber, Max, 1980 [1924]: Wirtschaft und Gesellschaft. Tübingen: J. C. B. Mohr (Paul Siebeck). White, Harrison, Scott A. Boorman und Ronald L. Breiger, 1976: Social Structure from Multiple Networks. I. Blockmodels of Roles and Positions, American Journal of Sociology 81, 4: 730780. 
Williamson, Oliver E., 1981: The Economics of Organization: The Transaction Cost Approach, American Journal of Sociology 87, 4: 548-577.

Zolberg, Aristide R., Astri Suhrke und Sergio Aguayo, 1989: Escape from Violence: Conflict and the Refugee Crisis in the Developing World. New York: Cambridge University Press. 
Tabellen und Schaubilder

Tabelle 1:

Globale Migration (stocks), 1960-2005 (in Millionen)

Year: $\begin{array}{llllllllll}1960 & 1965 & 1970 & 1975 & 1980 & 1985 & 1990 & 1995 & 2000 & 2005\end{array}$

$\underline{\text { Region }^{11}:}$

Welt

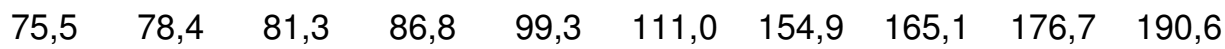

Entwickelte Regio-

nen

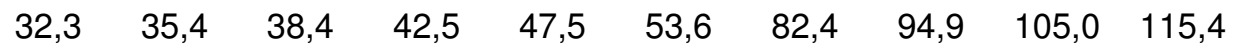

Weniger entwickelte

Regionen

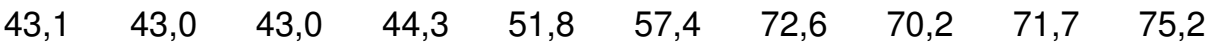

Am wenigsten entwickelte Länder

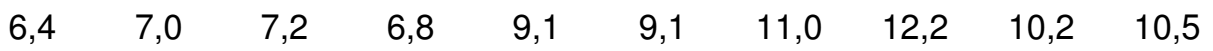

Quelle: Eigene Berechnungen (Prozentsätze) auf Basis von United Nations Secretariat 2005, Stand 14. November 2006; 8:37 Uhr.

11 "Region" bezieht sich hier nicht auf den Herkunftsort, sondern auf den Aufenthaltsort der Migranten zum jeweiligen Zeitpunkt. Die Grenzen einiger Regionen haben sich zwischen 1985 und 1990 verändert. Siehe dazu die Anmerkung der Population Division of the Department of Economic and Social Affairs of the United Nations Secretariat, Definition of major areas and regions, http://esa.un.org/migration/index.asp?panel=3 
Tabelle 2:

Globale Migration (stocks), 1960-2005 (Als Prozentsatz der Bevölkerung)

$\begin{array}{lllllllllll}\text { Year: } & 1960 & 1965 & 1970 & 1975 & 1980 & 1985 & 1990 & 1995 & 2000 & 2005\end{array}$

$\underline{\text { Region }^{12}:}$

Welt

$\begin{array}{llllllllll}2,5 & 2,3 & 2,2 & 2,1 & 2,2 & 2,3 & 2,9 & 2,9 & 2,9 & 2,9\end{array}$

Entwickelte Regio-

nen

$\begin{array}{llllllllll}3,4 & 3,5 & 3,6 & 3,9 & 4,2 & 4,6 & 7,2 & 8,1 & 8,8 & 9,5\end{array}$

Weniger entwickelte

Regionen

$\begin{array}{llllllllll}2,1 & 1,8 & 1,6 & 1,5 & 1,6 & 1,6 & 1,8 & 1,6 & 1,5 & 1,4\end{array}$

Am wenigsten ent-

wickelte Länder

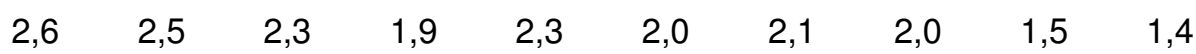

Quelle: Eigene Berechnungen (Prozentsätze) auf Basis von United Nations Secretariat 2005, Stand 14. November 2006; 8:37 Uhr.

${ }^{12}$ Siehe vorige Fußnote. 


\section{Schaubild 1:}

Eine stilisierte Migrationskurve - Allgemeine Diffusionskurve oder S-Kurve

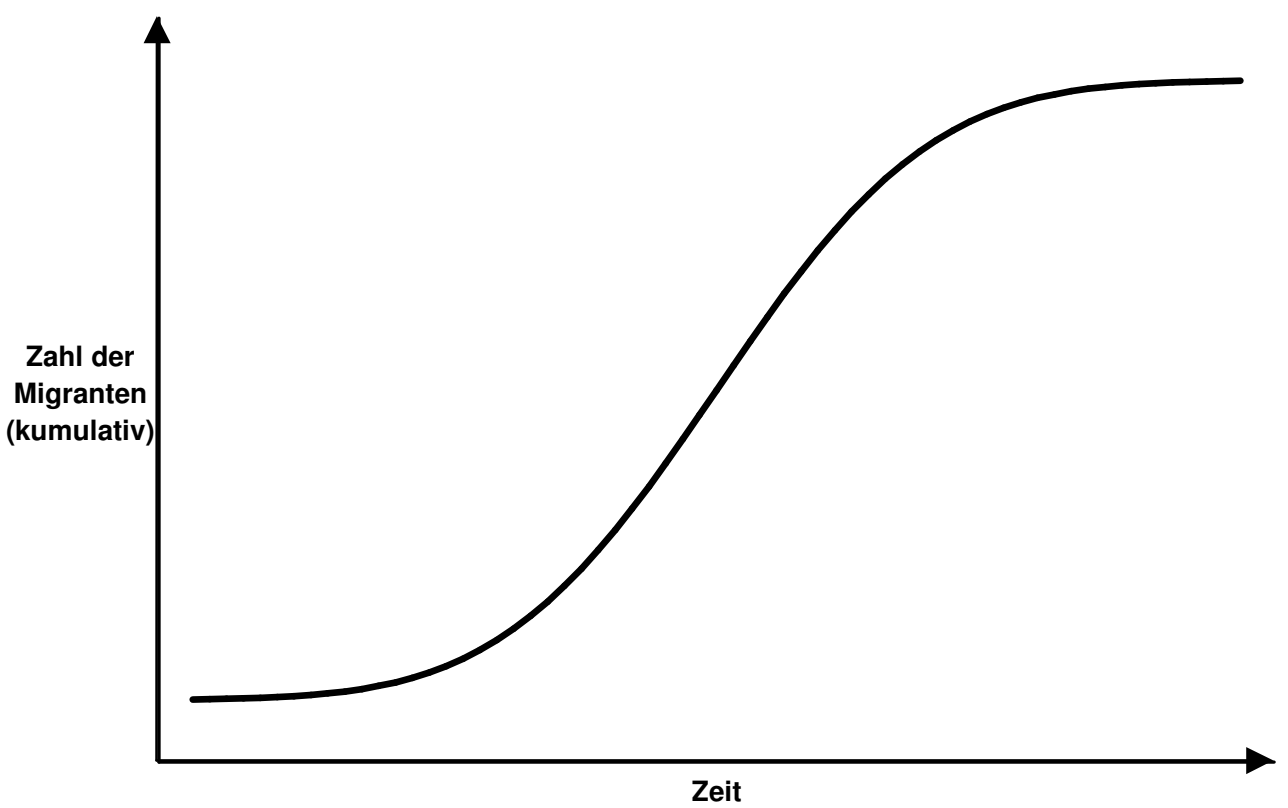

Quelle: Faist 2000a. 
Schaubild 2:

Unvollendete und unterbrochene türkisch-deutsche Migrationskurve (1960-2000)

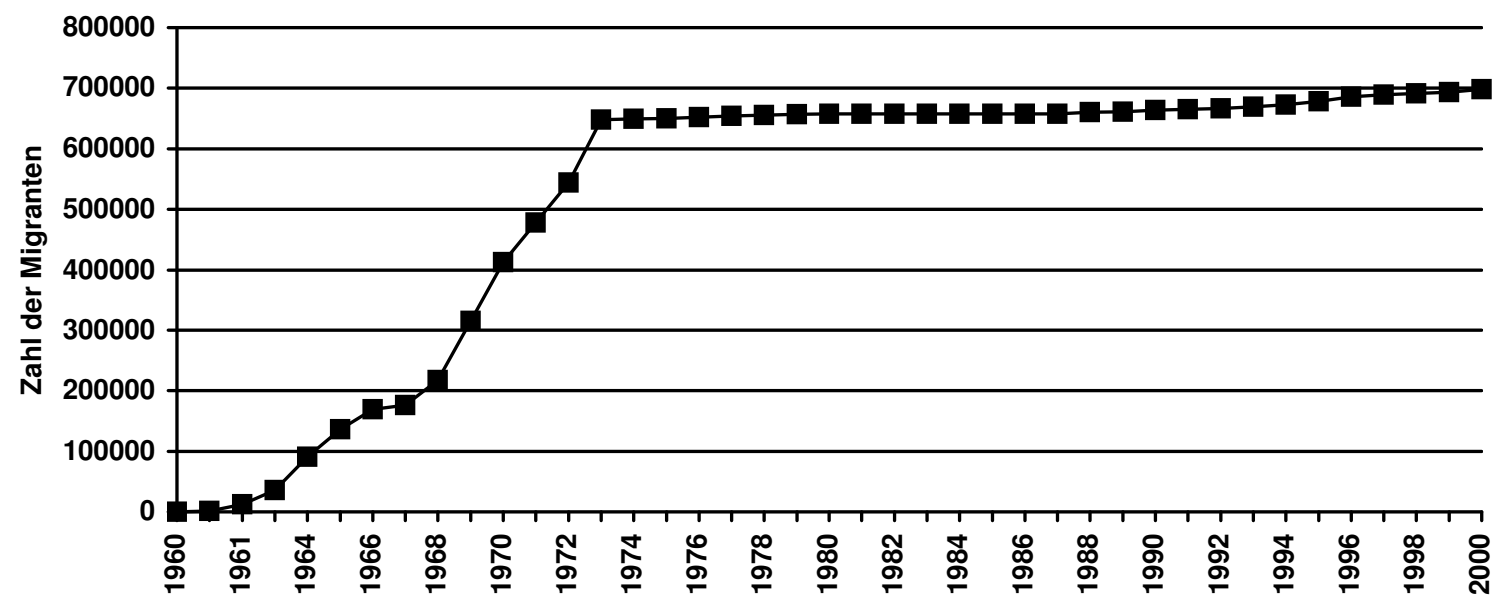

Quelle: Faist 2000a. 